Pedro Filipe Viana Ferreira Pinto ${ }^{1}$

Tiago José Santos Matos FerRaZ 2

Carla Maria Almeida Ramalho ${ }^{2}$

Nuno Aires Mota de Mendonça MONTENEGro ${ }^{3}$

Artigo Original

Palavras-chave

Corticosteroides/uso terapêutico Corticosteroides/administração \& dosagem Gravidez de alto risco Trabalho de parto prematuro Análise de sobrevida

Keywords

Adrenal cortex hormones/therapeutic use Adrenal cortex hormones/administration \& dosage Pregnancy, high risk Obstetric labor, premature Survival analysis

\section{Avaliação da ministração de corticosteroides em situações de suspeita de parto pré-termo iminente: um estudo de coorte retrospectivo em um centro terciário}

\author{
Evaluation of corticosteroid administration in situations of suspected \\ imminent preterm delivery: a retrospective cobort study in a tertiary centre
}

\section{Resumo}

OBJETIVO: A ministração antenatal de um ciclo único de corticoterapia está recomendada a mulheres grávidas entre a 24 e a 34 semanas com risco de parto prematuro. O efeito máximo é atingido quando os corticosteroides ministrados entre 24 horas e 7 dias antes do parto. $\bigcirc$ objetivo deste estudo foi avaliar a ocorrência de parto nos sete dias após corticoterapia nas principais situações obstétricas com risco de parto pré-termo. MÉTODOS: Estudo de coorte retrospectivo incluindo 209 grávidas internadas em risco de parto pré-termo submetidas a tratamento com corticosteroides para maturação pulmonar fetal. O estudo foi realizado entre janeiro de 2012 e março de 2014 e um hospital universitário. $\bigcirc$ desfecho principal avaliado foi o número de mulheres que tiveram parto no prazo de 7 dias após a ministração antenatal de corticosteroides. Foram definidos dois grupos de acordo com o motivo para iniciar corticosteroides: ameaça de parto prétermo (Grupo APPT) e outras indicações para corticoterapia (Grupo RPPT). Foi efetuada uma análise de sobrevivência de Kaplan-Meier e um valor $p<0,05$ foi considerado estatisticamente significativo. RESULTADOS: 46,4\% (n=97) das mulheres grávidas tiveram parto nos 7 dias após a ministração de corticosteroides. Parto em 7 dias ocorreu mais frequentemente no grupo RPPT, em comparação com o grupo APPT (57,3 versus 42,4\%, $p=0,001)$. Foi detectada uma diferença estatisticamente significativa entre a curva de sobrevivência para os grupos APPT e RPPT, com um hazard ratio para parto até 7 dias 1,71 vezes maior para o grupo RPPT (IC95\% 1,23-2,37; p<0,00 1). CONCLUSÃO: Pode-se concluir que a probabilidade de um evento (nascimento dentro de 7 dias após corticoterapia) é menor no grupo de grávidas internadas no contexto de ameaça de parto prétermo do que por outras indicações. A utilização de corticosteroides em grávidas internadas por suspeita de trabalho parto pré-termo deverá ser alvo de uma rigorosa avaliação clínica.

\section{Abstract}

PURPOSE: The administration of a single-course antenatal corticosteroid treatment is recommended for pregnant women between 24 and 34 weeks with risk of premature birth. The maximum effect is achieved when antenatal corticosteroids are administered between $24 \mathrm{~h}$ and 7 days before delivery. The objective of this study was to evaluate the occurrence of birth within seven days of corticosteroid therapy in major obstetric situations with risk of preterm birth. METHODS: Retrospective cohort study including 209 pregnant women hospitalized in risk of preterm delivery, submitted to corticosteroid therapy for fetal lung maturation. The study was carried out between January 2012 and March 2014 at a university hospital. Main outcome measure was the number of women who delivered within 7 da ys after antenatal corticosteroid administration. Two groups were defined according to the reason for starting corticosteroids: threatened preterm labour (Group APPT) and other indications for corticosteroid therapy (Group RPPT). A Kaplan-Meier survival analysis was performed and a p value $<0.05$ was considered statistically significant. RESULTS: $46.4 \%$ (n=97) of pregnant women gave birth in the seven days following corticosteroid administration. Delivery within 7 days occurred more frequently on group 2 in comparison to group 1 (57. 3 versus $42.4 \% ; p=0.001$ ). There is a statistically significant difference between the survival curve for groups 1 and 2, with a hazard ratio for delivery within 7 days 1.71 times higher for group 2 (95\% Cl 1.23-2.37; $p<0.001$ ). CONCLUSION: It can be concluded that the probability of an event (birth within 7 days after corticosteroids) is smaller in the group of pregnant women admitted in the context of threatened preterm labor than for other indications. The use of corticosteroids in pregnant women admitted for suspected preterm labor should be subject to rigorous clinical evaluation.

\section{Correspondência \\ Pedro Viana Pinto Alameda Prof. Hernâni Monteiro $4200-319$ \\ Porto, Portugal \\ Recebido \\ $07 / 05 / 2015$

Centro Hospitalar S. João - EPE - Portugal.

'Centro Hospitalar S. João - EPE - Porto, Portugal.

${ }^{2}$ Centro Hospitalar S. João - EPE - Porto, Portugal; Faculdade de Medicina, Universidade do Porto - Porto, Portugal; Instituto de Investigação e Inovação em Saúde, Universidade do Porto - Porto, Portugal.

${ }^{3}$ Centro Hospitalar S. João - EPE - Porto, Portugal; Faculdade de Medicina, Universidade do Porto - Porto, Portugal.

Conflito de interesses: não há 


\section{Introdução}

A prematuridade é o determinante isolado mais importante de desfecho adverso infantil, quer em termos de morbilidade, quer em termos de mortalidade ${ }^{1}$. Todos os anos, cerca de 1,1 milhão de recém-nascidos morrem como consequência de prematuridade, de acordo com estimativas realizadas em $2010^{2}$. Desde o primeiro ensaio randomizado realizado por Liggins e Howie, em $1972^{3}$, que a corticoterapia tem sido aplicada na população em risco de parto pré-termo. Aplicada corretamente, segundo uma meta-análise, a corticoterapia antenatal reveste-se de particular importância para diminuir a morbimortalidade associada à prematuridade, reduzindo a mortalidade neonatal (RR 0,69; IC95\% 0,58-0,81), bem como as incidências de síndrome de dificuldade respiratória (RR 0,66; IC95\% 0,59-0,73) e hemorragia cerebroventricular (RR 0,54; IC95\% 0,43-0,69), entre outros ${ }^{4}$. Atualmente, um ciclo único de corticosteroides (betametasona ou dexametasona) está recomendado a grávidas em risco de parto pré-termo entre a $24^{\mathrm{a}}$ e a $34^{\mathrm{a}}$ semana ${ }^{5,6}$.

A corticoterapia antenatal apresenta uma eficácia máxima nos primeiros sete dias após a sua ministração, sendo que após esse período os benefícios serão residuais ou até mesmo nulos ${ }^{4,7,8}$. Tendo em conta que a predição do risco de parto pré-termo imediato não é uma tarefa simples, um número significativo de mulheres será exposto sem necessidade a esse tipo de tratamento, enquanto outras poderão não receber o tratamento adequado (ou por ministração tardia ou por ausência de ministração do fármaco). Apesar de estudos de seguimento em longo prazo não terem documentado efeitos laterais major com a corticoterapia antenatal ${ }^{4,9}$, a real segurança desse tratamento tem sido questionada recentemente, especialmente em recém-nascidos que acabaram por nascer a termo. Estudos realizados em animais e estudos epidemiológicos questionam a segurança em longo prazo da exposição antenatal a corticosteroides, essencialmente por meio do conceito de programação fetal, que defende que a exposição a determinados fatores ambientais precocemente pode influenciar o desenvolvimento pré-natal e pode causar alterações estruturais e funcionais que persistem durante toda a vida ${ }^{10-15}$. Por outro lado, a realização de múltiplos ciclos de corticoterapia ou de ciclos de resgate mantém-se em debate, parecendo melhorar levemente os desfechos neonatais imediatos (morbimortalidade), mas sem alteração de desfechos em longo prazo e com possíveis efeitos laterais significativos, como restrição de crescimento fetal e alterações do desenvolvimento neurocognitivo, pelo que tais ciclos devem ser utilizados ainda com maior cautela ${ }^{16-22}$.

É, portanto, essencial a prescrição adequada de corticosteroides a mulheres grávidas, devendo ser feito um esforço para aplicar tal terapia apenas a mulheres que venham efetivamente a se beneficiar dela. O objetivo deste trabalho foi o de avaliar a ocorrência de parto nos sete dias após corticoterapia nas principais situações obstétricas com risco de parto pré-termo na nossa instituição.

\section{Métodos}

Foi realizado um estudo de coorte retrospetivo durante o período compreendido entre janeiro de 2012 e março de 2014 em uma instituição universitária com cuidados terciários. A população consistiu em grávidas expostas à corticoterapia para maturação pulmonar fetal. Todas as grávidas que receberam pelo menos uma dose de corticosteroides, quer $12 \mathrm{mg}$ de betametasona, quer $6 \mathrm{mg}$ de dexametasona, entre a $24^{a}$ e a $34^{a}$ semanas de gestação e com parto na nossa instituição, foram incluídas. As grávidas expostas foram identificadas após acesso a uma base de dados electrónica e ao sistema informático da nossa instituição $\left(\mathrm{OBSCARE}^{\circledR}, \mathrm{SCLINICO}^{\circledR}\right)$. Foram consultados os processos clínicos electrónicos individuais de forma a identificar as características gerais da nossa população, bem como o motivo de ministração dos corticoides e o tempo decorrido entre essa ministração e o parto. Foram excluídas gravidezes múltiplas, malformações fetais, casos de morte intrauterina e placentação anómala. Foram registadas as seguintes características: idade materna, paridade, índice de massa corporal (IMC) prévio à gravidez, idade gestacional aquando da ministração de corticosteroides, motivo para iniciar corticosteroide (ameaça parto pré-termo, rotura prematura de membranas, doença hipertensiva da gravidez, restrição crescimento fetal), idade gestacional do parto e tipo de parto. Foi definido parto pré-termo como parto antes da $37^{\mathrm{a}}$ semana.

Foi avaliada a proporção de grávidas com parto nos sete dias após ministração de corticosteroides, bem como o intervalo de tempo entre a ministração destes e o parto. Foram ainda comparados dois grupos de acordo com o motivo para iniciar corticosteroide: ameaça de parto pré-termo (Grupo APPT) e outras indicações com risco de parto pré-termo (Grupo RPPT). Não foi avaliada a proporção de grávidas com parto pré-termo sem exposição a corticosteroides, por não ser o objetivo do estudo, mas acreditamos que essa proporção será residual.

Os dados apresentados encontram-se sob a forma de números totais (n), proporções (\%) e médias/medianas com desvios padrão. Foi realizada uma análise de sobrevivência e utilizado o log rank test para comparação de curvas de sobrevivência, de acordo com o motivo para realizar corticoterapia. Foram construídas curvas de Kaplan-Meier para evidenciar o tempo desde a ministração de corticoide até o parto, de acordo com a indicação. $\mathrm{O}$ valor $\mathrm{p}<0,05$ foi considerado estatisticamente significativo. A análise estatística foi efetuada com o auxílio do software STATA. 
Resultados

Durante o período do estudo foram identificadas 236 grávidas submetidas à corticoterapia para maturação pulmonar fetal, sendo excluídas do estudo 17 mulheres (pelo seu parto ter ocorrido noutra instituição), sendo, portanto, analisadas 209 mulheres. As principais características da população em estudo encontram-se sumariadas na Tabela 1. O tempo médio até o parto desde a ministração de corticoides em toda a população foi de 8,9 dias (0-79). Não se detectou diferença significativa na probabilidade de parto em 7 dias entre nulíparas e multíparas $(p=0,9)$.

Das 209 grávidas submetidas à corticoterapia, $147(70,3 \%)$ tiveram um parto pré-termo e $97(46,4 \%)$ tiveram parto nos 7 dias seguintes à ministração desses fármacos. Quando comparados os dois grupos, as grávidas incluídas no Grupo RPPT (outras indicações com risco de parto pré-termo) apresentaram mais frequentemente um parto antes dos 7 dias que as grávidas incluídas no Grupo APPT (ameaça de parto pré-termo) - 57,3 versus $42,4 \%, p=0,001$. Existe ainda uma diferença significativa entre a curva de sobrevivência dos Grupos APPT e RPPT (Figura 1), com um hazard ratio para parto até

Tabela 1. Características gerais da população submetida à corticoterapia antenatal por risco de parto pré-termo

\begin{tabular}{|c|c|}
\hline Características maternas e parto & Total (n=209) \\
\hline Idade materna (média $\pm D P$ ) & $30,3 \pm 0,9$ \\
\hline Paridade - nulipara - n (\%) & $109(52)$ \\
\hline IMC (média $\pm D P)$ & $24,2 \pm 5,9$ \\
\hline Restrição crescimento fetal - n (\%) & 17 \\
\hline Doença hipertensiva da gravidez - n (\%) & 17 \\
\hline Idade gestacional parto mediana (mín.-máx.) & 34 sem (23-40 sem) \\
\hline Parto pré-termo - n (\%) & $147(70,3)$ \\
\hline Tipo de parto - cesariana - n (\%) & 41 \\
\hline
\end{tabular}

DP: desvio padrão; IMC: índice de massa corporal; sem: semanas.

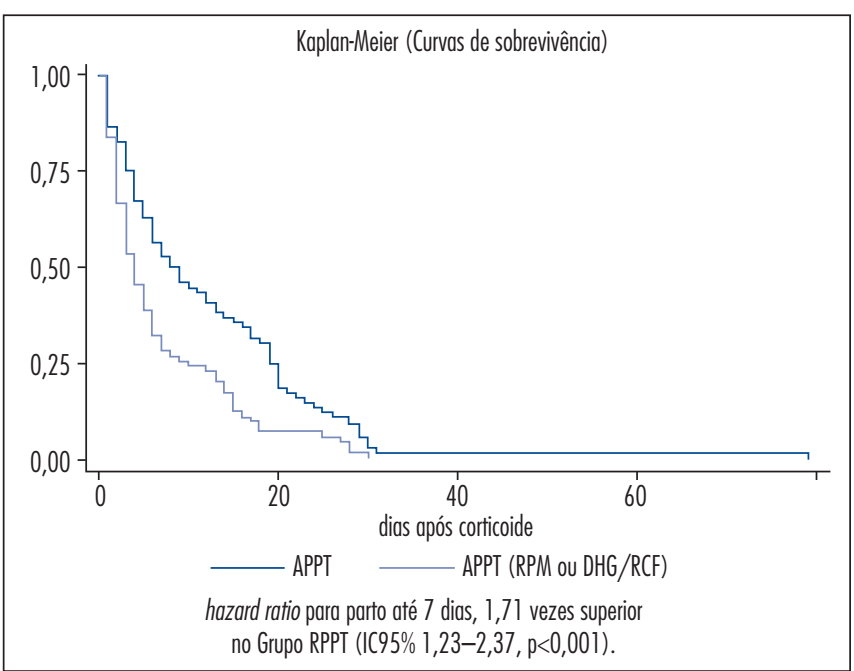

Figura 1. Intervalo de tempo desde a ministração de corticoides até o parto
7 dias 1,71 vezes superior no grupo de grávidas do Grupo RPPT (IC95\% 1,2-2,4; p<0,001).

\section{Discussão}

Neste estudo de coorte retrospetivo que avaliou a prescrição de corticosteroides na nossa instituição demonstramos que a percentagem de mulheres com idade gestacional compreendida entre a $24^{\mathrm{a}}$ e a $34^{\mathrm{a}}$ semana de gestação com parto nos primeiros 7 dias após ministração destes foi de $46,4 \%$; consequentemente, mais da metade das grávidas foi exposta a esse tratamento, sem contudo tirar benefício, podendo até mesmo ter sido prejudicadas.

Quando comparamos os dados por indicação para ministração de corticosteroides, verificamos que a probabilidade de parto em sete dias é menor no grupo de grávidas internadas por ameaça de parto pré-termo. Recentemente, foram publicados dois estudos que revelam resultados semelhantes ${ }^{23,24}$. Em uma análise por indicação para corticosteroide, a rotura prematura de membranas e as indicações maternas (essencialmente a doença hipertensiva da gravidez em todos os seus espectros) foram as indicações em que uma maior proporção de mulheres tiveram parto nos sete dias após ministração desses fármacos ${ }^{24}$.

O diagnóstico de ameaça de parto pré-termo reveste-se de uma enorme importância, embora seja igualmente difícil. De acordo com uma meta-análise recente, apenas cerca de $7 \%$ das mulheres com o diagnóstico de ameaça de parto pré-termo tiveram parto em 48 horas e $11 \%$ tiveram parto e uma semana ${ }^{25}$. Ferramentas como o teste de fibronectina fetal (com um excelente valor preditivo negativo, mas sensibilidade e valor preditivo positivo baixos) e a medição do colo uterino (um colo $>15 \mathrm{~mm}$ praticamente permite excluir um parto na próxima semana) permitem com maior segurança excluir esse diagnóstico. Dessa forma, a confiança nessas ferramentas torna possível diminuir o número de casos com ministração desnecessária desses fármacos ${ }^{25,26}$. Novos testes recentemente descritos aparentam ser promissores, com resultados superiores ao uso isolado do teste da fibronectina ${ }^{27}$.

Apesar de alguns dados favorecerem a segurança em longo prazo da corticoterapia antenatal ${ }^{9}$, alguns autores começam a questionar essa segurança, quer com base em estudos animais, quer em estudos em seres humanos. Embora aparentemente não tenham sido detectadas diferenças maiores de desenvolvimento neurocognitivo e hormonal e de risco cardiovascular ${ }^{4,9}$ em crianças e adultos expostos à corticoterapia antenatal, têm sido realçadas diferenças subtis em termos do funcionamento do eixo hipotálamo-hipofisário (com especial atenção 
para a amígdala e, consequentemente, para as emoções), alterações do desenvolvimento cerebral e da mielinização, níveis de cortisol sanguíneos, resistência à insulina e pressão arterial, entre outros ${ }^{9,10,14,15,28-31}$. De acordo com o conceito de programação fetal, considera-se que a exposição a um único ciclo de corticoterapia é insuficiente para, alterando o ambiente hormonal fetal, causar alterações definitivas a diversos sistemas, potencialmente via alteração dos padrões de metilação e acetilação de histonas envolvendo o receptor de glicocorticoides ${ }^{12,13}$. Tendo em conta que basta um ciclo de corticoterapia para poder desencadear as alterações responsáveis pela programação fetal, é de relevar que, no nosso estudo, mais da metade dos fetos foi exposta a um tratamento sem benefícios. Mais ainda, essas alterações poderão ser transmitidas à descendência ${ }^{32}$. Mais estudos serão necessários para compreender melhor a segurança em longo prazo da utilização desses fármacos.

Torna-se preocupante a generalização da utilização da corticoterapia antenatal. O estudo de Polyakov, na Austrália, demonstra um aumento gradual da exposição desnecessária de fetos a corticosteroides ao longo do período em estudo, dados confirmados recentemente no estudo de Razaz conduzido no Canadá33,34. A assunção da sua inocuidade com a dificuldade de diagnóstico das verdadeiras situações de parto pré-termo iminente levou à generalização da sua utilização, a ponto de haver mulheres que recebem múltiplos ciclos de corticoterapia (com evidência de benefícios ligeiros, mas ainda sem garantia de segurança em longo prazo e com um claro aumento de risco de restrição de crescimento fetal $)^{16-22}$ fazendo corticoterapia após as 35 semanas de gestação (apesar de haver plausibilidade biológica para tal utilização, não estão provados cientificamente os benefícios ou os riscos da corticoterapia nestas situações $)^{35-39}$ e ainda submetidas à corticoterapia em ambulatório. Dados resultantes do Multiple Courses of Antenatal Corticosteroids for Preterm Birth Study (MACS) apontam para um maior risco de alterações neurocognitivas aos cinco anos de idade em crianças expostas a múltiplos ciclos de corticoterapia e com parto a termo ${ }^{22}$. É importante não se esquecer de exemplos recentes, como a utilização de antibioterapia na ameaça de parto pré-termo sem ruptura de membranas com um aumento do risco de alterações neurocognitivas e paralisia cerebral nas crianças expostas ${ }^{40,41}$. Outra questão importante é a da aplicabilidade da corticoterapia em países em desenvolvimento: um estudo recente não conseguiu encontrar benefício na utilização de corticoides em fetos em risco de parto pré-termo, tendo inclusive detectado um aumento da mortalidade em fetos que nasceram a termo ${ }^{42}$.

Uma limitação do estudo é a sua natureza retrospetiva. Por um lado, não foi possível compreender qual a motivação para ministração do fármaco naquela altura em particular; por outro lado, os critérios de diagnóstico das condições estudadas poderão não ter sido aplicados de forma uniforme pelos profissionais envolvidos, apesar da existência de protocolos de serviço para atuação nas referidas situações. Também, o número de casos não permitiu efetuar uma análise discriminada por indicação para corticoterapia, permitindo apenas a comparação entre os dois grupos definidos (ameaça de parto pré-termo versus outras indicações). Durante o período de tempo em estudo foram ministrados dois tipos diferentes de corticosteroides. No entanto, dado que o objetivo do estudo não visava a avaliação da sua eficácia neonatal e tendo em conta que, apesar de reais, são ligeiras as diferenças entre a betametasona e a dexametasona em termos de eficácia e farmacocinética, tal fato não parece constituir uma limitação para a análise efetuada ${ }^{6,43}$.

A corticoterapia antenatal é uma das principais armas disponíveis para diminuir a morbimortalidade neonatal, principalmente em um grupo de tão grande risco de complicações imediatas e em longo prazo como os prematuros. Contudo, a exposição desnecessária de fetos a esse tratamento deve ser evitada, principalmente com um desconhecimento ainda grande de potenciais efeitos laterais em longo prazo. Na nossa população a probabilidade de parto em 7 dias foi maior para o grupo 2 (outras indicações) que para a ameaça de parto pré-termo. É, pois, essencial refinar o diagnóstico dessa identidade, bem como o timing da ministração de corticosteroides. Idealmente, a corticoterapia deverá ser aplicada apenas a fetos que venham a se beneficiar de tal tratamento, devendo ser minimizada a exposição desnecessária a esses fármacos.

\section{Referências}

1. Chang $\mathrm{HH}$, Larson J, Blencowe $\mathrm{H}$, Spong $\mathrm{CY}$, Howson $\mathrm{CP}$, CairnsSmith $S$, et al. Preventing preterm births: analysis of trends and potential reductions with interventions in 39 countries with very high human development index. Lancet. 2013;381 (9862):223-34.
2. Liu L, Johnson HL, Cousens S, Perin J, Scott S, Lawn JE, et al. Global, regional, and national causes of child mortality: an updated systematic analysis for 2010 with time trends since 2000 . Lancet. 2012;379(9832):2151-61. 
3. Liggins GC, Howie RN. A controlled trial of antepartum glucocorticoid treatment for prevention of the respiratory distress syndrome in premature infants. Pediatrics. 1972;50(4):515-25.

4. Roberts D, Dalziel S. Antenatal corticosteroids for accelerating fetal lung maturation for women at risk of preterm birth. Cochrane Database Syst Rev. 2006;(3):CD004454.

5. Royal College of Obstetricians \& Gynaecologists [Internet]. Antenatal corticosteroids to reduce neonatal morbidity and mortality. 2010. (Green-top Guideline No. 7) [cited 2015 Jan 20]. Available from: https://www.rcog.org.uk/globalassets/ documents/guidelines/gtg_7.pdf

6. Wapner R, Jobe AH. Controversy: antenatal steroids. Clin Perinatol. $2011 ; 38(3): 529-45$.

7. McEvoy C, Schilling D, Spitale P, Peters D, O'Malley J, Durand M. Decreased respiratory compliance in infants less than or equal to 32 weeks' gestation, delivered more than 7 days after antenatal steroid therapy. Pediatrics. 2008;121(5):e1032-8.

8. Waters TP, Mercer B. Impact of timing of antenatal corticosteroid exposure on neonatal outcomes. J Matern Fetal Neonatal Med. 2009;22(4):311-4.

9. Dalziel SR, Walker NK, Parag V, Mantell C, Rea HH, Rodgers A, et al. Cardiovascular risk factors after antenatal exposure to betamethasone: 30-year follow-up of a randomised controlled trial. Lancet. 2005;365(9474): 1856-62.

10. Doyle LW, Ford GW, Davis NM, Callanan C. Antenatal corticosteroid therapy and blood pressure at 14 years of age in preterm children. Clin Sci (Lond). 2000;98(2):137-42.

11. Seckl JR. Prenatal glucocorticoids and long-term programming. Eur J Endocrinol. 2004;151 Suppl 3:U49-62.

12. Harris A, Seckl J. Glucocorticoids, prenatal stress and the programming of disease. Horm Behav. 2011 ;59(3):279-89.

13. Sandman CA, Davis EP, Buss C, Glynn LM. Prenatal programming of human neurological function. Int J Pept. $2011 ; 2011: 837596$.

14. Alexander N, Rosenlocher F, Stalder T, Linke J, Distler W, Morgner $J$, et al. Impact of antenatal synthetic glucocorticoid exposure on endocrine stress reactivity in term-born children. J Clin Endocrinol Metab. 2012;97(10):3538-44.

15. Waffarn F, Davis EP. Effects of antenatal corticosteroids on the hypothalamic-pituitary-adrenocortical axis of the fetus and newborn: experimental findings and clinical considerations. Am J Obstet Gynecol. 2012;207(6):446-54.

16. Aghajafari F, Murphy K, Matthews S, Ohlsson A, Amankwah K, Hannah M. Repeated doses of antenatal corticosteroids in animals: a systematic review. Am J Obstet Gynecol. 2002;186(4):843-9.

17. French NP, Hagan R, Evans SF, Mullan A, Newnham JP. Repeated antenatal corticosteroids: effects on cerebral palsy and childhood behavior. Am J Obstet Gynecol. 2004; 190(3):588-95.

18. Murphy KE, Hannah ME, Willan AR, Hewson SA, Ohlsson A, Kelly EN, et al. Multiple courses of antenatal corticosteroids for preterm birth (MACS): a randomised controlled trial. Lancet. 2008;372(9656):2143-51.

19. Asztalos EV, Murphy KE, Hannah ME, Willan AR, Matthews SG, Ohlsson A, et al. Multiple courses of antenatal corticosteroids for preterm birth study: 2-year outcomes. Pediatrics. 2010;126(5):e1045-55.

20. Peltoniemi OM, Kari MA, Hallman M. Repeated antenatal corticosteroid treatment: a systematic review and meta-analysis. Acta Obstet Gynecol Scand. 2011 ;90(7):719-27.

21. McKinlay CJ, Crowther CA, Middleton P, Harding JE. Repeat antenatal glucocorticoids for women at risk of preterm birth: a Cochrane Systematic Review. Am J Obstet Gynecol. 2012;206(3): 187-94.
22. Asztalos EV, Murphy KE, Willan AR, Matthews SG, Ohlsson A, Saigal S, et al. Multiple courses of antenatal corticosteroids for preterm birth study: outcomes in children at 5 years of age (MACS5). JAMA Pediatr. 2013;167(12): $1102-10$.

23. Vis JY, Wilms FF, Kuin RA, Reuvers JM, Stam MC, Pattinaja DA, et al. Time to delivery after the first course of antenatal corticosteroids: a cohort study. Am J Perinatol. $2011 ; 28(9): 683-8$.

24. Boesveld M, Heida KY, Oudijk MA, Brouwers HA, Koenen SV, Kwee A. Evaluation of antenatal corticosteroid prescribing patterns among 984 women at risk for preterm delivery. J Matern Fetal Neonatal Med. 2014;27(5):516-9.

25. Sotiriadis A, Papatheodorou S, Kavvadias A, Makrydimas G. Transvaginal cervical length measurement for prediction of preterm birth in women with threatened preterm labor: a meta-analysis. Ultrasound Obstet Gynecol. 2010;35(1):54-64.

26. Sanchez-Ramos L, Delke I, Zamora J, Kaunitz AM. Fetal fibronectin as a short-term predictor of preterm birth in symptomatic patients: a meta-analysis. Obstet Gynecol. 2009;1 14(3):63 1-40.

27. Liong S, Di Quinzio MK, Fleming G, Permezel M, Rice GE, Georgiou HM. New biomarkers for the prediction of spontaneous preterm labour in symptomatic pregnant women: a comparison with fetal fibronectin. BJOG. 2014;122(3):370-9.

28. Fletcher AJ, McGarrigle HH, Edwards CM, Fowden AL, Giussani DA. Effects of low dose dexamethasone treatment on basal cardiovascular and endocrine function in fetal sheep during late gestation. J Physiol. 2002;545(Pt 2):649-60.

29. Davis EP, Waffarn F, Uy C, Hobel CJ, Glynn LM, Sandman CA. Effect of prenatal glucocorticoid treatment on size at birth among infants born at term gestation. J Perinatol. 2009;29(11):731-7.

30. Berry $\mathrm{M}$, Jaquiery AL, Oliver MH, Harding JE, Bloomfield FH. Antenatal corticosteroid exposure at term increases adult adiposity: an experimental study in sheep. Acta Obstet Gynecol Scand. 2013;92(7):862-5.

31. Pettit KE, Tran SH, Lee E, Caughey AB. The association of antenatal corticosteroids with neonatal hypoglycemia and hyperbilirubinemia. J Matern Fetal Neonatal Med. 2014;27(7):683-6.

32. Crudo A, Petropoulos S, Moisiadis VG, lqbal M, Kostaki A, Machnes $Z$, et al. Prenatal synthetic glucocorticoid treatment changes DNA methylation states in male organ systems: multigenerational effects. Endocrinology. 2012;153(7):3269-83.

33. Polyakov A, Cohen S, Baum M, Trickey D, Jolley D, Wallace EM. Patterns of antenatal corticosteroid prescribing 1998-2004. Aust N Z J Obstet Gynaecol. 2007;47(1):42-5.

34. Razaz N, Skoll A, Fahey J, Allen VM, Joseph KS. Trends in optimal, suboptimal, and questionably appropriate receipt of antenatal corticosteroid prophylaxis. Obstet Gynecol. $2015 ; 125(2): 288-96$.

35. Venkatesh VC, Katzberg HD. Glucocorticoid regulation of epithelial sodium channel genes in human fetal lung. Am J Physiol. 1997;273/1 Pt 1):L227-33.

36. Jain L, Chen XJ, Ramosevac S, Brown LA, Eaton DC. Expression of highly selective sodium channels in alveolar type II cells is determined by culture conditions. Am J Physiol Lung Cell Mol Physiol. $2001 ; 280(4)$ :L646-58.

37. Jain L, Dudell GG. Respiratory transition in infants delivered by cesarean section. Semin Perinatol. 2006;30(5):296-304.

38. Porto AM, Coutinho IC, Correia JB, Amorim MM. Effectiveness of antenatal corticosteroids in reducing respiratory disorders in late preterm infants: randomised clinical trial. BMJ. $2011 ; 342:$ d 1696. 
39. Kamath-Rayne BD, DeFranco EA, Marcotte MP. Antenatal steroids for treatment of fetal lung immaturity after 34 weeks of gestation: an evaluation of neonatal outcomes. Obstet Gynecol. 2012;119(5):909-16.

40. Kenyon S, Pike K, Jones DR, Brocklehurst P, Marlow N, Salt A, et al. Childhood outcomes after prescription of antibiotics to pregnant women with spontaneous preterm labour: 7-year follow-up of the ORACLE II trial. Lancet. 2008;372(9646):1319-27.

41. Flenady V, Hawley G, Stock OM, Kenyon S, Badawi N. Prophylactic antibiotics for inhibiting preterm labour with intact membranes. Cochrane Database Syst Rev. 2013;12:CD000246.
42. Althabe F, Belizán JM, McClure EM, Hemingway-Foday J, Berrueta M, Mazzoni A, et al. A population-based, multifaceted strategy to implement antenatal corticosteroid treatment versus standard care for the reduction of neonatal mortality due to preterm birth in low-income and middleincome countries: the ACT cluster-randomised trial. Lancet. 2015;385(9968):629-39.

43. Brownfoot FC, Gagliardi DI, Bain E, Middleton P, Crowther CA. Different corticosteroids and regimens for accelerating fetal lung maturation for women at risk of preterm birth. Cochrane Database Syst Rev. 2013;8:CD006764. 